\title{
Intracellular and Surface Acetylcholine Receptors During the Normal Development of a Frog Skeletal Muscle
}

\author{
J. Goldfarb, C. Cantin, and M. W. Cohen \\ Department of Physiology, McGill University, Montreal, Quebec H3G 1Y6, Canada
}

${ }^{125} \mid-\alpha$-bungarotoxin $\left({ }^{125} \mid-\alpha \mathrm{BT}\right)$ was used to measure the pool sizes of surface and intracellular acetylcholine receptors (AChRs) in the myotomal muscle of Xenopus laevis over a developmental period (stages 23-48; 1.03-7.5 d) which ranged from initial to mature stages of neuromuscular synaptogenesis. The surface pool increased progressively throughout development. The intracellular pool increased more slowly and also underwent a transient decrease. Linear regression indicated that $\mathrm{AChRs}$ begin to appear intracellularly and in the surface membrane at embryonic ages of 13.2 and $18.5 \mathrm{hr}$, respectively. The findings also suggest that newly synthesized AChRs contribute much more to the intracellular pool than do AChRs internalized from the surface membrane and that the rates of supply and/or intracellular resident times of these 2 sources of intracellular AChRs change during the course of normal development.

Carbamylcholine, even at concentrations 10 -fold greater than needed to block completely ${ }^{125} \mid-\alpha B T$ binding to surface AChRs, blocked specific intracellular binding by only $80 \%$. Considered in the light of previous studies on cell cultures, these results suggest that $20 \%$ of the intracellular sites are on $\alpha$-subunits not yet assembled into pentameric AChRs.

Light microscope radioautography revealed an essentially uniform distribution of intracellular AChRs along the length of the muscle cells. It is concluded that during the normal development of Xenopus myotomal muscle the accumulation and maintenance of AChRs in the postsynaptic membrane occurs in the absence of any preferential concentration of intracellular AChRs in the subsynaptic region.

Ncuromuscular synaptogencsis is accompanicd by marked changes in the number, distribution, and density of acetylcholine receptors (AChRs) in the surface membrane of muscle cells. Just before the arrival of the growing motor axons, surface AChRs are distributed at relatively low density along the entire length of the developing muscle cells. With the onset of synaptogenesis, AChRs accumulate rapidly in the newly-forming postsynaptic membrane, leading to a much greater density there than in extrasynaptic regions (Bevan and Steinbach, 1977; Burden, 1977; Harris, 1981; Chow and Cohen, 1983). As synaptogenesis progresses, the density of extrasynaptic AChRs declines but muscle

\footnotetext{
Received Apr. 25, 1989; revised Aug. 14, 1989; accepted Aug. 17, 1989.

We thank G. Hébert for expert technical assistance and M. Quik for gifts of $\alpha$-bungarotoxin. This work was supported by the Medical Research Council of Canada.

Correspondence should be addressed to Dr. M. W. Cohen, Department of Physiology, McGill University, 3655 Drumnond Streel, Montreal, Quebec H3G 1Y6, Canada.

Copyright $@ 1990$ Society for Neuroscience $0270-6474 / 90 / 100500-08 \$ 02.00 / 0$
}

growth continues, so that the total number of surface AChRs increases in some muscles and decreases transiently in others (Burden, 1977; Betz et al., 1980; Chow and Cohen, 1983). Thereafter, as the muscle and its synapses continue to grow, the number of surface AChRs increases further (Burden, 1977; Betz et al., 1980; Steinbach, 1981; Chow and Cohen, 1983).

Changes also occur in the number and distribution of intracellular AChRs during neuromuscular synaptogenesis, but the available information in this regard is relatively sparse. In one study on rat muscle, limited to ages when the extrasynaptic density of surface AChRs was at its mature low values, it was found that the intracellular AChRs were restricted to the synaptic region and did not extend more than $300 \mu \mathrm{m}$ beyond the synaptic sites (Pestronk, 1985). This distribution is consistent with recent findings indicating that $\mathrm{mRNA}$ for $\mathrm{AChR}$ subunits is likewise restricted to synaptic regions at such ages (Merlie and Sanes, 1985; Fontaine et al., 1988). Another study examined the distribution of intracellular AChRs in chick muscle at earlier stages, when surface AChRs are preferentially localized at synaptic sites but are still present extrasynaptically at densities greater than the mature low value (Atsumi, 1981). The results revealed the presence of intracellular $A C h R s$ along the entire length of the muscle cells but were ambiguous concerning whether synaptic regions have higher levels of intracellular AChRs at these stages. In the absence of additional studies, developmental changes in the number and distribution of intracellular AChRs and their relationship to developmental changes in surface AChRs remain largely unknown for the major period of neuromuscular synaptogenesis.

In the present study we have measured the pool sizes of surface and intracellular AChRs in the myotomal muscle of the frog Xenopus laevis, from a few hours after the onset of innervation to stages at which neuromuscular synaptogenesis is at or close to maturity. We have also compared the binding properties of the AChRs in these 2 pools and examined the distribution of the intracellular AChRs. Some of the findings were reported previously in abstract form (Goldfarb et al., 1984).

\section{Materials and Methods}

Animals and dissections. Embryos of Xenopus laevis were obtained by induced breeding and reared in fresh dechlorinated tap water at room temperature $\left(22-24^{\circ} \mathrm{C}\right)$. They developed at about the same rate as described by Nieuwkoop and Faber (1967) and hatched after $2 \mathrm{~d}$. Beginning on the fourth day, the tadpoles were fed finely ground frog brittle.

For each experiment, animals of the same developmental stage were selected according to the external criteria of Nieuwkoop and Faber (1967). Their range of lengths never exceeded $10 \%$ of the mean length of the group. Throughout the text the ages for each developmental stage are based on those published by Nieuwkoop and Faber (1967).

"Tail preparations," consisting of the myotomes and their associated 


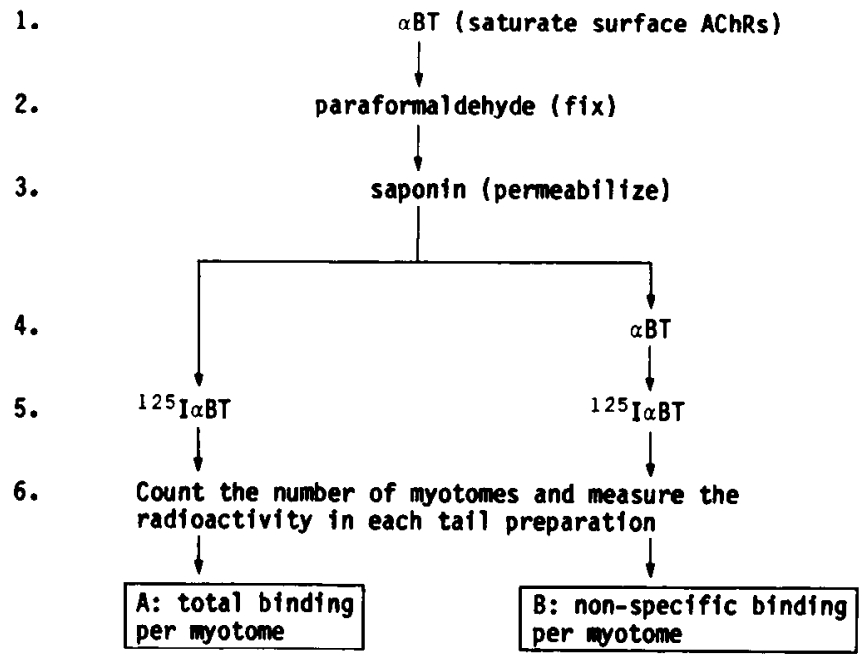

specific binding per myotome $=A-B$

Figure 1. Experimental protocol for measuring the binding of ${ }^{125} \mathrm{I}-\alpha \mathrm{BT}$ to intracellular AChRs. At least 8 tail preparations of the same stage were used in each experiment. After step 3, they were separated into 2 equal groups in order to determine total binding per myotome $(A)$ and nonspecific binding per myotome $(B)$. Specific binding per myotome was obtained by subtracting $B$ from $A$.

spinal cord and notochord, were isolated from anesthetized animals as described previously (Chow and Cohen, 1983). Excluded from these tail preparations were the smaller, more caudal, myotomes as well as the first 2-4 rostral myotomes. As a result, the tail preparations contained myotomes which were approximately similar in size. The $\mathrm{pH}$ of the anesthetic (tricaine methanesulfonate) was always adjusted to 7.2-7.4 as described previously (Cohen et al., 1984).

To restrict development during the dissection period, tail preparations were placed in ice-cold Ringer's until all dissections were completed. The Ringer's solution had the following composition: $\mathrm{NaCl}, 111 \mathrm{~mm}$; $\mathrm{KCl}, 3 \mathrm{~mm}$; $\mathrm{CaCl}_{2}, 1.8 \mathrm{~mm}$; HEPES buffer adjusted to $\mathrm{pH} 7.4$ with $\mathrm{NaOH}, 10 \mathrm{~mm} ; 0.5 \%(\mathrm{vol} / \mathrm{vol})$ dialyzed horse serum (Grand Island Biological Co.). The serum reduced the "stickiness" of the tail preparations as well as nonspecific binding of ${ }^{125} \mathrm{I}-\alpha$-bungarotoxin ( ${ }^{125} \mathrm{I}-\alpha \mathrm{BT}$ ). Except where stated otherwise, all procedures were carried out at room temperature.
1:I- $\alpha B$ T binding to intracellular AChRs. In order to detect intracellular AChRs, a modified version of the protocol originally described by Fambrough and Devreotes (1978) was used. The procedure is illustrated in Figure 1. Tail preparations ( 8 or more) were exposed to $\alpha$ BT (2-4 $\mu \mathrm{g} / \mathrm{ml}$ ) for 30-60 min to saturate all surface AChRs (step 1) and then rinsed several times for $1 \mathrm{hr}$ with refrigerated Ringer's to remove unbound $\alpha \mathrm{BT}$. They were then fixed with paraformaldehyde (step 2). In some experiments, steps 1 and 2 were reversed, without any obvious effect on the results. The fixative was added in a series of increasing concentrations over $5 \mathrm{~min}$ to prevent contraction. Following this initial fixation, the tail preparations were immersed in $1-4 \%$ paraformaldehyde, usually for $3 \mathrm{hr}$ at $4^{\circ} \mathrm{C}$ but in some cases for $1 \mathrm{hr}$ at room temperature. The higher concentration provided better preservation, especially at younger stages, but did not substantially alter binding of ${ }^{125} \mathrm{I}-\alpha \mathrm{BT}$ to AChRs (see Results). The paraformaldehyde fixative was based on the formulation of McLean and Nakane (1974) and had the following composition: paraformaldehyde, $1-4 \%$ (wt/vol); lysine, 75 $\mathrm{mM}$; sodium phosphate, $38 \mathrm{~mm}$; sodium $m$-periodate, $10 \mathrm{~mm}$; dextrose, $56 \mathrm{~mm}$. $\mathrm{NaOH}$ was added to adjust the $\mathrm{pH}$ to $\sim 7.2$. Following fixation, the tail preparations were rinsed several times with Ringer's, and in some experiments they were then stored overnight in the refrigerator.

Next, the tail preparations were permeabilized by treatment with saponin ( $5 \mathrm{mg} / \mathrm{ml}$ in Ringer's) for $30 \mathrm{~min}$ (step 3). They were then rinsed for $30 \mathrm{~min}$ and divided into 2 equal groups, one of which was treated with $\alpha$ BT (2-4 $\mu \mathrm{g} / \mathrm{ml}$ in Ringer's) for 30-60 min to saturate intracellular AChRs (step 4) so that nonspecific binding could be assessed. Then, both groups were exposed to ${ }^{125} \mathrm{I}-\alpha \mathrm{BT}(0.3-1.1 \mu \mathrm{g} / \mathrm{ml}$ in Ringer's) for $60 \mathrm{~min}$ (step 5). The concentration varied with the batch of ${ }^{125} \mathrm{I}-\alpha \mathrm{BT}$ and was tested as described below to ensure saturation of the AChRs. Following several rinses, the tail preparations were stored overnight in the refrigerator.

In some experiments, a third group of tail preparations was exposed to carbamylcholine chloride (CARB) instead of $\alpha \mathrm{BT}$ in step 4. In these cases, the same concentration ( $1.6 \mathrm{~mm}$ or greater) of this reversible ligand was present in all solutions from steps 4-6 for this group of tail preparations.

After exposure to ${ }^{125} \mathrm{I}-\alpha \mathrm{BT}$ and overnight rinsing, the tail preparations were trimmed at their ends to remove the previously cut myotomes. The number of remaining myotomes was counted, and each tail preparation was placed in a plastic vial for measurement of radioactivity in a gamma counter (Nuclear Chicago, model 1185). Background values were also measured, and subtracted, so that the amount of bound ${ }^{125} \mathrm{I}$ $\alpha \mathrm{BT}$ per myotome could be calculated. Specific binding per myotome was estimated as the difference between the 2 groups (Fig. 1).

12: I- $\alpha B T$ binding to surface AChRs and to all AChRs. To examine binding of ${ }^{125} \mathrm{I}-\alpha \mathrm{BT}$ to surface AChRs, steps $1-3$ were omitted from the experimental protocol. Step 2 was included in those experiments where prefixation was desired. To examine binding of ${ }^{125} \mathrm{I}-\alpha \mathrm{BT}$ to all AChRs
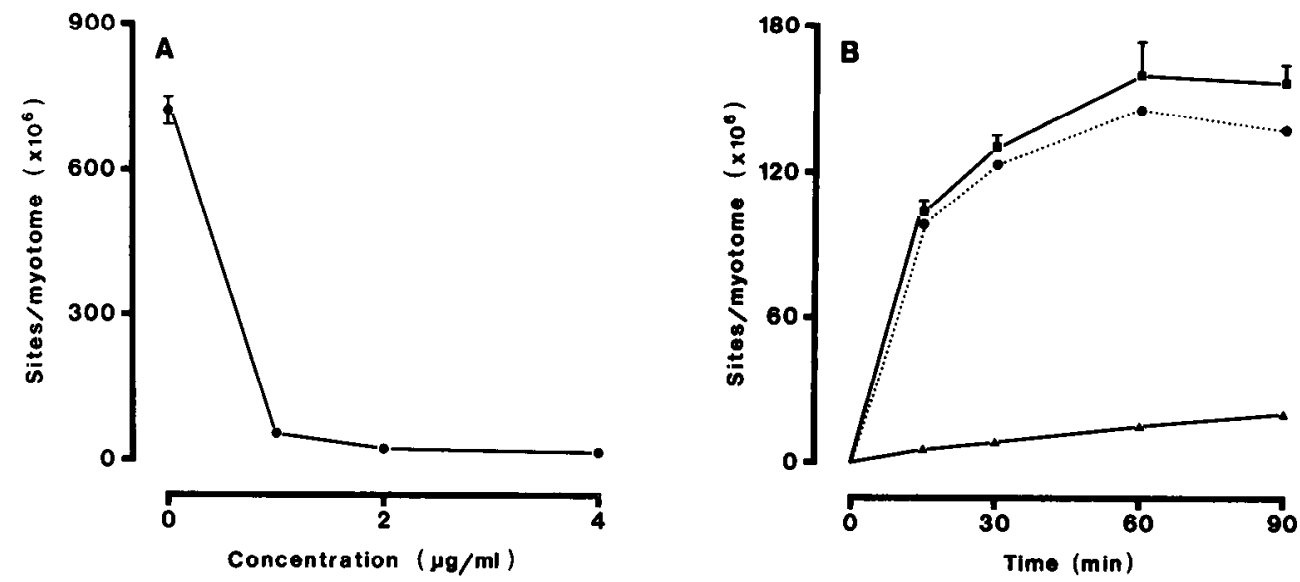

Figure 2. Saturation of AChRs with $\alpha \mathrm{BT}$ and with ${ }^{125} \mathrm{I}-\alpha \mathrm{BT}$. $A$, Effectiveness of $\alpha \mathrm{BT}$ in blocking surface binding of ${ }^{125} \mathrm{I}-\alpha \mathrm{BT}$. Stage 48 (7.5 d) tail preparations were exposed for $30 \mathrm{~min}$ to $\alpha \mathrm{BT}$ at the indicated concentrations and then to $0.6 \mu \mathrm{g} / \mathrm{ml}$. ${ }^{125} \mathrm{I}-\alpha \mathrm{BT}$ for $60 \mathrm{~min}$. Means and standard error bars (where possible to show) are based on 4 tail preparations. Note that saturation of surface AChRs was achieved at $2 \mu \mathrm{g} / \mathrm{ml} \alpha \mathrm{BT}$. $B$, Effectiveness of ${ }^{125} \mathrm{I}-\alpha \mathrm{BT}$ in saturating intracellular AChRs. Total (squares), nonspecific (triangles), and specific (circles) binding were determined in stage $46(4.4 \mathrm{~d})$ tail preparations exposed to $0.3 \mu \mathrm{g} / \mathrm{ml}{ }^{125} \mathrm{I}-\alpha \mathrm{BT}$ for the times indicated on the abscissa. Each value of total and nonspecific binding is based on 5 tail preparations. Note that saturation of intracellular AChRs with ${ }^{125}$ I- $\alpha$ BT was achieved in 60 min. 

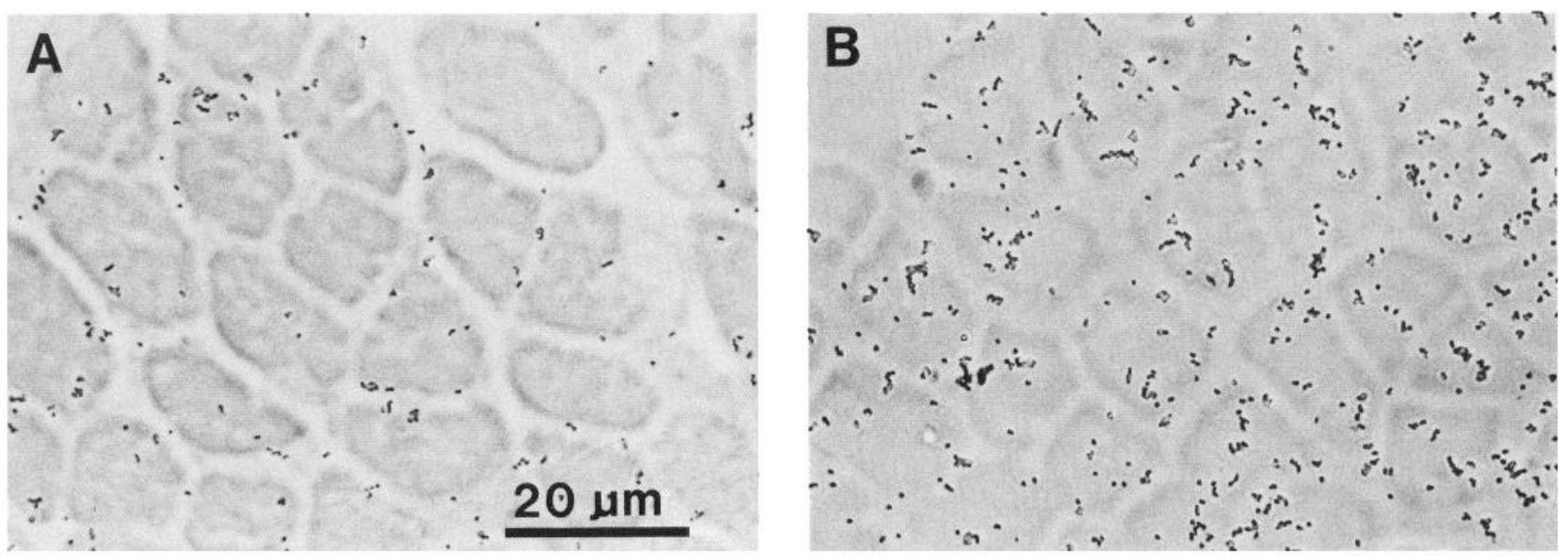

Figure 3. Radioautographs of transverse sections of myotomal muscle. A, Stage $46(4.4 \mathrm{~d})$ tail preparation processed to reveal surface AChRs. $B$, Stage $45(4.1 \mathrm{~d})$ tail preparation processed to reveal intracellular AChRs. Note that in $A$ the grains are predominantly located at the periphery of the muscle cells, whereas in $B$ they are distributed throughout the muscle cells, thereby indicating that treatment with saponin was effective in exposing intracellular AChRs. Same scale for $A$ and $B$.

(both surface and intracellular), step 1 was omitted from the experimental protocol.

Saturation of AChRs by $\alpha B T$ and ${ }^{125} I-\alpha B T$. The potency of each new batch of $\alpha \mathrm{BT}$ (kindly provided by Dr. M. Quik) and of ${ }^{125} \mathrm{I}-\alpha \mathrm{BT}$ (New England Nuclear) was tested by measuring ${ }^{125} \mathrm{I}-\alpha \mathrm{BT}$ binding after exposing groups of tail preparations (stage 46 or greater) to different concentrations of toxin for the same time and/or to the same concentration of toxin for different times (Fig. 2). At least 4 tail preparations were used for each concentration and time tested. On the basis of these tests, $2-4 \mu \mathrm{g} / \mathrm{ml} \alpha \mathrm{BT}$ for $30-60 \mathrm{~min}$ was found to be effective in saturating intracellular and surface AChRs (Fig. $2 A$ ). The batches of ${ }^{125} \mathrm{I}-\alpha \mathrm{BT}$ were usually more potent. For most batches, surfaces AChRs and intracellular AChRs were saturated within $60 \mathrm{~min}$ when the tail preparations were immersed in $0.3-0.4 \mu \mathrm{g} / \mathrm{ml}{ }^{125} \mathrm{I}-\alpha$ BT (Fig. $2 B$ ). Occasional batches of ${ }^{125} \mathrm{I}-\alpha \mathrm{BT}$ had to be used at a concentration of $0.6-0.7 \mu \mathrm{g} / \mathrm{ml}$ for $60 \mathrm{~min}$ or $1.0-1.1 \mu \mathrm{g} / \mathrm{ml}$ for $60 \mathrm{~min}$ to ensure saturation of the AChRs.

Radioautography. Tail preparations which had already been exposed to ${ }^{125} \mathrm{I}-\alpha \mathrm{BT}$ were postfixed for $1 \mathrm{hr}$ with $1.5 \%(\mathrm{vol} / \mathrm{vol})$ glutaraldehyde (in $0.1 \mathrm{~m}$ phosphate buffer, $\mathrm{pH}$ 7.3). After rinsing, trimming, counting their myotomes, and measuring their radioactivity, the tail preparations were dehydrated, embedded in Epon 812, and cut longitudinally or transversely. The $1 \mu \mathrm{m}$ sections were mounted on microscope slides and processed for radioautography as described previously (Chow and Cohen, 1983). When the grain density over myotomal muscle cells of test slides reached acceptable levels $\left(200-500 / 2500 \mu \mathrm{m}^{2}\right)$, all the remaining slides were processed together.

Grains were counted at a magnification of $1250 \times$ by viewing them through a Zeiss microscope fitted with an appropriate ocular grid. Sites were chosen only where the cells were well preserved and clearly resolved with phase-contrast optics. The microscope was then focused on the grains, and the counting was done using bright-field optics. Each value of grain density presented in the Results is based on 40 measurements in each of 3-4 tail preparations. Background grain densities, measured in 20 different regions without tissue, were typically $10-15 / 2500 \mu \mathrm{m}^{2}$ and were subtracted.

\section{Results}

Assessment of experimental protocol

Effect of fixation on ${ }^{125} I-\alpha B T$ binding. Fixation with $1 \%$ paraformaldehyde barely affected specific binding of ${ }^{125} \mathrm{I}-\alpha \mathrm{BT}$ to surface AChRs, whether assessed with $\alpha$ BT (fixed/unfixed: 0.95 \pm 0.03 , mean \pm SEM for 5 experiments) or with CARB (fixed/ unfixed: $0.94 \pm 0.04, n=4)$. In 5 additional experiments, specific binding after fixation with $4 \%$ paraformaldehyde was 97 $\pm 3 \%$ of the value for $1 \%$ paraformaldehyde. Thus, in Xenopus tail preparations, as in cultures of embryonic chick muscle cells
(Fambrough and Devreotes, 1978), the great majority of AChRs retain their capacity to bind cholinergic ligands following fixation with paraformaldehyde.

Permeabilization with saponin. The effectiveness of saponin in exposing intracellular AChRs was revealed by radioautography. In the absence of treatment with saponin, the grains tended to be limited to the periphery of the muscle cells (Fig. $3 A$ ), whereas after treatment with saponin, the grains were distributed throughout the muscle cells (Fig. $3 B$ ).

Specific intracellular binding of ${ }^{125} \mathrm{I}-\alpha \mathrm{BT}$ (1) was virtually unaffected $(101 \pm 11 \%, n=3)$ when the concentration of saponin was reduced 5-fold, (2) was decreased slightly to 83 and $95 \%$ in 2 experiments in which saponin was present in all solutions from steps 3-6 in Figure 1, and (3) was essentially unaltered (95 and $104 \%$ ) in 2 experiments in which the rinse time was extended 6 times longer than normal. These results suggest that our standard treatment with saponin was optimal for exposing intracellular AChRs to ${ }^{125} \mathrm{I}-\alpha \mathrm{BT}$ and that the permeabilization was irreversible.

To determine whether saponin interferes with the binding of ${ }^{125}$ I- $\alpha$ BT to AChRs, 24-30 tail preparations were fixed and then divided into 3 groups. The first group was used to measure ${ }^{125} \mathrm{I}-$ $\alpha \mathrm{BT}$ binding to surface AChRs and was not treated with saponin. The second and third groups were used to measure ${ }^{125} \mathrm{I}-$ $\alpha \mathrm{BT}$ binding to intracellular AChRs and to all AChRs (both surface and intracellular) and were treated with saponin. The arithmetic difference in specific binding between the second and third groups gives an estimate of the binding to surface AChRs after treatment with saponin. In 3 such experiments, this value was $112 \pm 10 \%$ of that obtained without saponin treatment. It follows that treatment with saponin did not interfere with binding of ${ }^{125} \mathrm{I}-\alpha \mathrm{BT}$ to AChRs.

An additional conclusion suggested by the above experiments is that saponin did not extract AChRs. This conclusion was tested more directly by measuring specific surface binding in fixed tail preparations, then treating the same tail preparations with saponin and measuring them again. The postsaponin value was $99 \pm 1 \%(n=17)$ of that prior to treatment with saponin.

${ }^{125} I-\alpha B T$ binding to different tissues. Measurements of grain density and relative tissue volume in radioautographs of trans- 


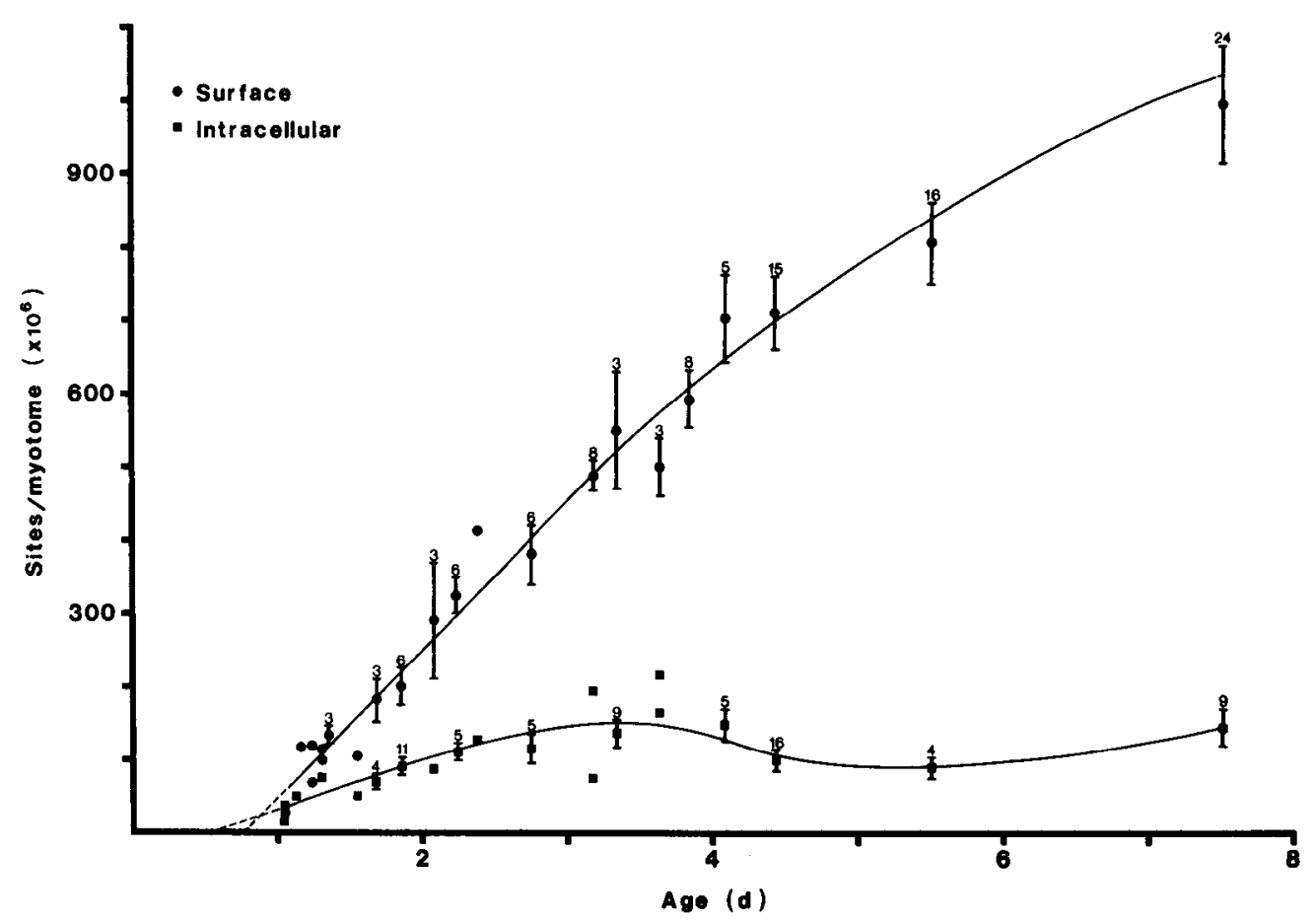

Figure 4. Developmental changes in the size of the surface pool (circles) and intracellular pool (squares) of AChRs. Stages have been converted to equivalent ages as determined by Nieuwkoop and Faber (1967). In cases where 3 or more experiments were made on the same stage, the values are given as means and standard errors and the number of experiments is indicated. For surface AChRs, the smooth curve is based on linear regression up to stage $42(3.3 \mathrm{~d})$ and extrapolates to an age of $18.5 \mathrm{hr}$ for their first appearance. For intracellular AChRs the smooth curve is based on lincar regression up to stage $32(1.9 \mathrm{~d})$ and extrapolates to an age of $13.2 \mathrm{hr}$ for their first appearance. verse sections of tail preparations at stages 31,40 , and 45 (1.6, 2.8 , and $4.1 \mathrm{~d}$, respectively) indicated that $<1 \%$ of the intracellular binding was associated with spinal cord and notochord. This is in agreement with the finding that mRNA transcripts for AChR subunits in Xenopus embryos are restricted to the myotomal muscle (Baldwin et al., 1988). In view of these findings, it is unnecessary to free the myotomes from the spinal cord and notochord prior to gamma counting (see Chow and Cohen, 1983), and as a result losses of muscle cells are eliminated.

\section{Developmental changes in the number of $A C h R s$}

Specific binding of ${ }^{125} \mathrm{I}-\alpha \mathrm{BT}$ to surface $\mathrm{AChRs}$ and to intracellular AChRs was measured between stages 23-48. Stage 23 (1.03 d) is only $\sim 5 \mathrm{hr}$ after the onset of innervation of the developing myotomal muscle (Kullberg et al., 1977; Chow and Cohen, 1983). By stage 48 ( $7.5 \mathrm{~d}$ ), myotomal muscle development and neuromuscular synaptogenesis are at or close to maturity as judged by electron microscopy, the channel properties and distribution of the surface AChRs, the short-lasting synaptic currents (Kullberg et al., 1977, 1980, 1985; Chow and Cohen, 1983; Kullberg and Kasprzak, 1985), and the relatively long half-life of the surface AChRs (see Discussion).

Changes in the size of the surface pool of AChRs are summarized in Figure 4. It can be seen that the number of specific binding sites per myotome increased progressively throughout development and reached a value of $10^{\circ}$ by stage 48 (7.5 d). Based on linear regression, the rate of accumulation up to stage 42 (3.3 d) was $205 \times 10^{6} \mathrm{sites} / \mathrm{myotome} / \mathrm{d}(n=45, r=0.90)$. Thereafter, the rate of accumulation slowed but was still as high as $100 \times 10^{6}$ sites/myotome/d between stages $47-48$ (5.5-7.5 d). Extrapolation of the linear relationship at the earlier stages yielded an embryonic age of $18.5 \mathrm{hr}$ at which specific binding sites begin to appear in the surface membrane. This value is $\sim 1$ hr less than was estimated previously (Chow and Cohen, 1983).

As shown in Figure 4, the number of specific intracellular binding sites per myotome also increased during the earlier stages of development but leveled off at a value of $\sim 150 \times 10^{6}$ by stages $41-45(3.2-4.1 \mathrm{~d})$. Thereafter, it declined to $\sim 100 \times$ $10^{6}$ at stages $46-47(4.4-5.5 \mathrm{~d})$ and returned to $\sim 150 \times 10^{6}$ by stage $48(7.5 \mathrm{~d})$. Based on linear regression, the rate of accumulation up to stage $32(1.9 \mathrm{~d})$ was $69 \times 10^{6}$ sites/myotome/d ( $n=20, r=0.68$ ), about one-third the rate for surface sites during the same developmental period. Extrapolation yielded an embryonic age of $13.2 \mathrm{hr}$ for the first appearance of intracellular sites. This estimate, considered with our estimate of the first appearance of surface AChRs, suggests that the intracellular resident time of newly synthesized AChRs is $\sim 5 \mathrm{hr}$ at these early stages.

\section{Inhibition of ${ }^{125} I-\alpha B T$ binding by a small cholinergic ligand}

To assess whether CARB is effective in blocking ${ }^{125} \mathrm{I}-\alpha \mathrm{BT}$ binding to intracellular AChRs, tail preparations were separated into 3 equal groups. Two of the groups were used as usual to measure (A) total binding and (B) nonspecific binding after treatment with $\alpha$ BT (Fig. 1). The third group was used to measure (C) nonspecific binding in the presence of CARB by substituting CARB for $\alpha$ BT in step 4 of the protocol (Fig. 1) and maintaining it in all solutions thereafter. In this way, 2 estimates of specific binding were obtained, one based on $\alpha \mathrm{BT}(\mathrm{A}-\mathrm{B})$, as in the previous section, and the other based on CARB $(\mathrm{A}-\mathrm{C})$.

As summarized in Figure 5, estimates of specific binding of ${ }^{125}$ I- $\alpha$ BT to surface AChRs were essentially the same whether based on $\alpha \mathrm{BT}$ or $1.6 \mathrm{~mm}$ CARB. This was the case even when the tail preparations were prefixed with paraformaldehyde (shaded column) or pretreated with both paraformaldehyde and saponin (open column). By contrast, specific intracellular binding based on CARB was only $75-80 \%$ of that based on $\alpha \mathrm{BT}$, even when the concentration of CARB was increased 10-fold (Fig. 5). These reduced values were obtained throughout development $(76 \pm 2 \%$ for eight stage $24-34$ experiments and 77 $\pm 3 \%$ for eight stage $45-48$ experiments). 


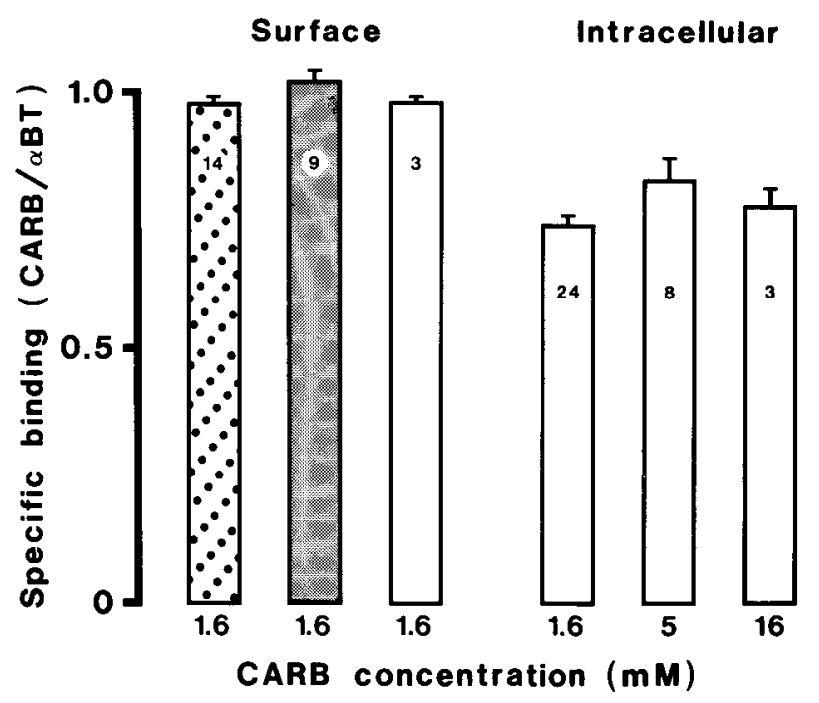

Figure 5. Estimates of specific ${ }^{125} \mathrm{I}-\alpha \mathrm{BT}$ binding based on CARB and on $\alpha \mathrm{BT}$. In each experiment, 2 estimates of specific binding were obtained. The one based on CARB is shown relative to the one based on $\omega B$. Note that for surface AChRs both estimates were the same whether examined in unfixed tail preparations (dotted column) or in tail preparations pretreated with paraformaldehyde (shaded column) or with paraformaldehyde and saponin (open column). By contrast, for intracellular AChRs the estimates based on CARB were only $75-80 \%$ of those based on $\alpha \mathrm{BT}$, even when the concentration of CARB was increased 10 -fold. The number of experiments on which each mean and SEM is based is shown within each column.

The above results could be explained in 2 ways: either CARB fails to block $\sim 20 \%$ of the specific intracellular binding sites or it generates additional nonspecific binding sites intracellularly. The latter possibility was excluded by the finding, in each of 2 experiments, that nonspecific binding of ${ }^{125} \mathrm{I}-\alpha \mathrm{BT}$ was not increased when tail preparations were exposed to CARB after blocking intracellular AChRs with $\alpha \mathrm{BT}$. Taken altogether, the results indicate that CARB can block only $\sim 80 \%$ of the specific intracellular ${ }^{125} \mathrm{I}-\alpha \mathrm{BT}$ binding sites, even when used at a concentration which is 10 -fold greater than needed to block entirely ${ }^{125} \mathrm{I}-\alpha \mathrm{BT}$ binding to surface AChRs.

\section{Distribution of intracellular AChRs}

The distribution of intracellular AChRs was examined by radioautography in longitudinal sections of myotomal muscle at stages 27 (1.3 d), 33/34 (1.9 d), and 45 (4.1 d). Examples are shown in Figure 6. In all cases, the grain distribution appeared to be relatively uniform along the length of the muscle cells, except for lower densities over the centrally located nuclei and over the yolk granules that are present in the muscle cells at early stages of development. There was no apparent increase at the ends of the muscle cells, the region of dense innervation (Lewis and Hughes, 1960; Kullberg et al., 1977; Chow and Cohen, 1983). Measurements of grain density confirmed that there were no statistically significant differences $(p \geq 0.3)$ at and away from the ends of the muscle cells at each of the 3 stages examined (Fig. 7). At stage 45, when yolk granules are no longer present, measurements were also made in successive $5 \times 5 \mu \mathrm{m}$ squares, beginning at the ends of the muscle cells and continuing beyond their midpoint. The successive values, based on 40 muscle cells in each of 3 tail preparations, fluctuated by less than $30 \%$, thereby indicating further the essentially uniform distribution of grains along the entire length of the muscle cells. By contrast, in ra- dioautographs of the surface AChRs there is a very marked elevation of grain density at the ends of the muscle cells by stage 33/34 (Chow and Cohen, 1983).

\section{Discussion}

In this study we have compared the number of surface and intracellular AChRs in Xenopus myotomal muscle, from within a few hours after the onset of innervation to relatively mature stages of neuromuscular synaptogenesis. Our data indicate that while the surface pool continues to enlarge throughout this period of normal development, the intracellular pool undergoes more complex changes in size. Below we discuss the likely factors which contribute to these changes and consider 2 additional main findings: the different binding properties of the surface and intracellular AChRs and the essentially uniform distribution of intracellular AChRs along the length of the muscle cells.

\section{Number, origin, and resident times of intracellular AChRs}

The fact that the number of surface AChRs continues to increase at a relatively high rate throughout development (Fig. 4) means that the rate of insertion of AChRs into the surface membrane must exceed the rate of internalization of surface AChRs. Except for the period where the pool of intracellular AChRs actually declines, the rate of AChR synthesis must be even greater than the rate of insertion. Accordingly, for much, if not all, of the developmental period we examined, the rate of synthesis exceeds the rate of internalization. In addition, studies on cultures of embryonic chick muscle cells have indicated that the intracellular resident time of newly synthesized AChRs is about an order of magnitude greater than that of the internalized AChRs (Devreotes and Fambrough, 1976; Fambrough et al., 1977). It follows from these considerations that during the normal development of Xenopus myotomal muscle, newly synthesized AChRs contribute much more to the intracellular pool than do surface-derived AChRs.

Our estimate that the synthesis of AChRs begins at an embryonic age of $13.2 \mathrm{hr}$ (Fig. 4) correlates well with the finding that mRNA transcripts for AChR subunits are detectable in Xenopus embryos as young as $13.75 \mathrm{hr}$ (Baldwin et al., 1988). Given an embryonic age of $18.5 \mathrm{hr}$ at which AChRs begin to appear in the surface membrane (Fig. 4), it follows that at these early stages there is a $5 \mathrm{hr}$ interval between the synthesis of an $\mathrm{AChR}$ and its insertion into the surface membrane. More direct measurements in primary cultures of embryonic chick muscle cells (Devreotes et al., 1977; Gardner and Fambrough, 1979) and nerve cells (Stollberg and Berg, 1987) have yielded a corresponding value of $2-3 \mathrm{hr}$, possibly reflecting a homeothermpoikilotherm difference.

According to the measurements of Baldwin et al. (1988), the myotomal levels of mRNA transcripts for the $\alpha$ - and $\delta$-subunits of the AChR remain more or less stable between stages 25-42 (1.15-3.2 d). If the rate of AChR synthesis is proportional to these mRNA levels, then it too should be stable during these stages. In the absence of any other changes, the intracellular pool size would be expected to reach a steady state within a few hours after stage 25 depcnding on the actual intracellular resident time of the newly synthesized AChRs. However, the pool size continues to increase for at least $2 \mathrm{~d}$ beyond stage 25 (Fig. 4). At least 3 factors may be responsible. The rising numbers of surface $A C h R s$ should result in an increasing rate of supply of internalized AChRs, thereby increasing the contribution of surface-derived AChRs to the intracellular pool. Any increase 

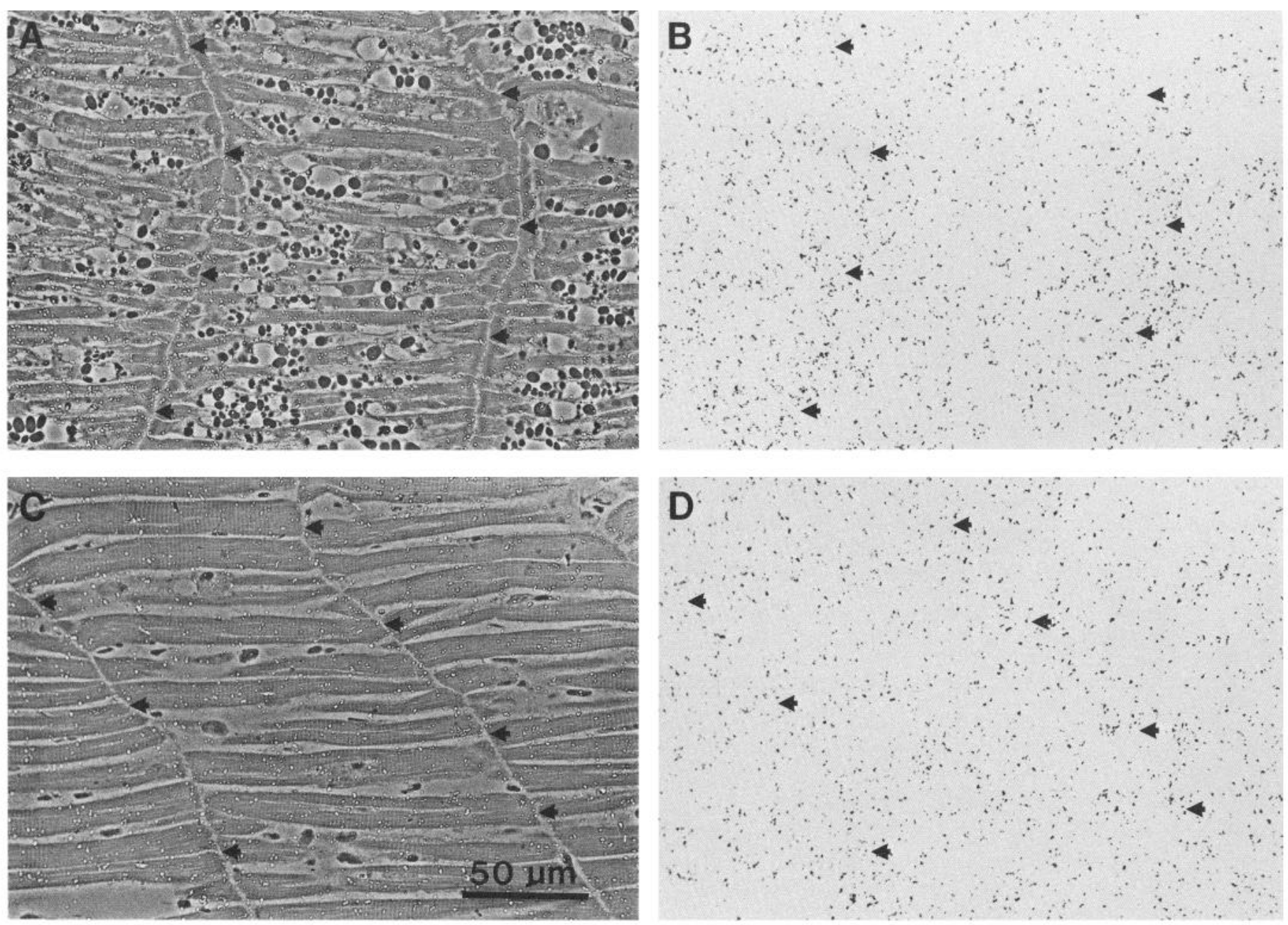

Figure 6. Radioautographs of intracellular ${ }^{125} \mathrm{I}-\alpha \mathrm{BT}$ binding in myotomal muscle. Phase contrast and corresponding bright-field views are shown. $A$ and $B$, Stage $33 / 34(1.9 \mathrm{~d})$. $C$ and $D$, Stage 45 (4.1 d). The muscle cells are oriented horizontally and their ends are marked by the phase-bright intermyotomal junctions (arrowheads). Note that the grains appear to be distributed randomly. Yolk granules are present in the muscle cells at stage $33 / 34$ but not at stage 45 . The scale in $C$ applies to the entire figure.

in the intracellular resident time of newly synthesized AChRs would also contribute to the increase in intracellular pool size. In addition, the rate of synthesis may increase beyond stage 25 despite unchanging levels of mRNA for the $\alpha$ - and $\delta$-subunits of the AChR.

Even though the intracellular pool derives its AChRs from 2 sources, its rate of increase up to stage $33 / 34$ (1.9 d) was only one-third that of the surface pool (Fig. 4). Furthermore, the surface pool continues to increase at a relatively high rate throughout development, even at those stages where the size of intracellular pool is relatively stable or decreasing. A key factor in explaining these differences is the resident times of AChRs in both pools. As discussed above, the intracellular resident time of the newly synthesized AChRs is only a few hours, and the value for internalized AChRs is probably much less. By contrast, measurements of the degradation of the surface AChRs in Xenopus tail preparations indicate that the mean half-life of the AChRs in the surface membrane is $\sim 60 \mathrm{hr}$ up to stage 35 / 36 (2.1 d) and reaches $\sim 140 \mathrm{hr}$ during the next $3 \mathrm{~d}$ (unpublished observations). By remaining in the surface membrane for such long times, many more newly synthesized AChRs can accumulate there in a given time than can accumulate intracellularly in the same time. Accordingly, the surface pool increases more rapidly than the intracellular pool, and it can continue to increase even while the intracellular pool is decreasing.

Beginning at $\sim 3.5 \mathrm{~d}$, there is a transient decline in the size of the intracellular pool (Fig. 4). At about the same time, the slope of the relationship between surface pool size and developmental age begins to decrease as well (Fig. 4), suggesting that a decreased rate of AChR synthesis may account for the decline in the intracellular pool. Subsequently, between 5.5-7.5 d, the size of the intracellular pool increases again but without an apparent increase in the rate of accumulation of AChRs in the surface membrane. A possible explanation for this result is that it arises from an enhanced rate of supply of surface-derived AChRs or from an increase in the intracellular resident times of the AChRs.

\section{Binding properties of intracellular AChRs}

When CARB was used at a concentration which inhibited ${ }^{125} \mathrm{I}$ $\alpha \mathrm{BT}$ binding to surface AChRs as completely as $\alpha \mathrm{BT}$, it inhibited only $75 \%$ of the specific intracellular sites that were sensitive to $\alpha \mathrm{BT}$. Even when the CARB concentration was increased 10fold, the inhibition was only $\sim 80 \%$ of that by $\alpha \mathrm{BT}$. Thus, unlike 


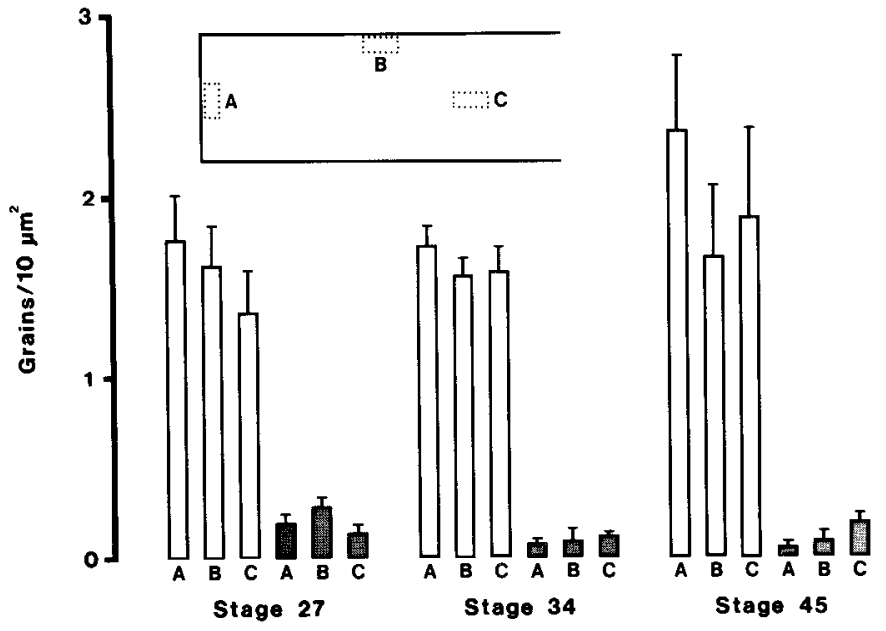

Figure 7. Intracellular grain densities at different sites. Grains were counted within $2 \times 5 \mu \mathrm{m}$ rectangles at 3 different sites. $A$, The first 2 $\mu \mathrm{m}$ below the cell surface at the ends of the muscle cells, the major site of innervation. $B$, The first $2 \mu \mathrm{m}$ below the cell surface, at least $10 \mu \mathrm{m}$ away from the ends of the muscle cells. Innervation is rare at such sites. $C$, The central core of the muscle cell, at least $10 \mu \mathrm{m}$ away from the cell end. Open columns indicate total intracellular binding, and shaded columns indicate nonspecific binding. Note that the grain densities at subsynaptic sites $(A)$ and at nonsynaptic sites $(B, C)$ were similar. Means and standard errors are based on 3-4 tail preparations. In each preparation, 40 measurements were made at each site. Grain densities were normalized to those of stage 45 , based on the specific activity of the ${ }^{125} \mathrm{I}-\alpha \mathrm{BT}$ and the time that the radioautographs were developed (Matthcws-Bellinger and Salpeter, 1978). The correction factors were 0.78 for stage 27 and 1.22 for stage 34 .

surface AChRs, all of which can bind CARB, some $20 \%$ of the specific intracellular sites appear to be unable to bind CARB.

This finding can be explained most simply in terms of what is known about the acquisition of cholinergic binding properties during the synthesis of the AChR in cultures of embryonic muscle cells and cell lines (Carlin et al., 1986; Merlie and Smith, 1986; Blount and Merlie, 1988). These studies have indicated that as it proceeds along the synthetic pathway, the $\alpha$-subunit acquires the capacity to bind $\alpha \mathrm{BT}$ even though it has little if any affinity for small cholinergic ligands such as CARB. Highaffinity binding for these ligands is acquired shortly afterwards when the individual subunits join to form the pentameric AChRs. Therefore, in Xenopus myotomal muscle those $20 \%$ of the specific intracellular toxin sites which do not bind CARB may be on unassembled $\alpha$-subunits on route to being synthesized into AChRs. This is consistent with the conclusion that in Xenopus myotomal muscle, the intracellular pool is derived mainly from newly synthesized AChRs.

\section{Distribution of intracellular AChRs}

During the developmental period between stage $27(1.3 \mathrm{~d})$ and stage $33 / 34(1.9 \mathrm{~d})$, there is a large preferential accumulation of surface AChRs at the ends of the myotomal muscle cells, the site of dense innervation (Chow and Cohen, 1983). Yet at neither of these stages was the distribution of intracellular AChRs significantly increased in this region (Fig. 7). An essentially uniform distribution was also observed at stage 45 (4.1 d) when the myotomal neuromuscular junction is relatively mature as judged by synaptic current duration (Kullberg et al., 1980, 1985), AChR channel open time (Kullberg and Kasprzak, 1985), and the half-life of surface AChRs (unpublished observations). Ap- parently, neither the development nor the maintenance of the high density of AChRs in the postsynaptic membrane of Xenopus myotomal muscle is associated with a preferential accumulation of intracellular AChRs subsynaptically.

It is of interest to consider these findings in terms of the 2 sources of intracellular AChRs. At all 3 stages examined, surface AChRs are present not only at sites of innervation but also along the entire length of the muscle cells (Chow and Cohen, 1983). Accordingly, surface-derived intracellular AChRs must originate along the entire length of the muscle cell. Although surface AChRs are present at highest density at sites of innervation, internalization at these sites would not necessarily be that much greater than in extrasynaptic regions if the synaptic AChRs have a substantially greater half-life than the extrasynaptic ones. Rapid movement away from the site of internalization would also contribute to a uniform distribution of surface-derived intracellular AChRs.

Newly synthesized AChRs are the major source of intracellular AChRs in Xenopus myotomal muscle. Exactly where they are synthesized along the length of the muscle cell is not known. In a recent study on muscles from 15 -d-old chicks it was found that mRNA for the $\alpha$-subunit of the AChR is localized at subsynaptic nuclei (Fontaine et al., 1988). Xenopus myotomal muscle cells have a single, centrally located nucleus at least until stage 46 (4.4 d; Muntz, 1975). The fact that the distribution of intracellular AChRs was not elevated in the midregion of the muscle cells or elsewhere along their length suggests either that synthesis is widely distributed throughout the cell or that, if it is localized to a specific region of the cell, the newly formed AChRs are transported rapidly away from that site. Interestingly, even in embryonic chick muscle at stages when there is rapid preferential accumulation of $\mathrm{AChRs}$ in the postsynaptic membrane (Atsumi, 1981) and in rat muscle at later stages (Pestronk, 1985), the intracellular AChRs extend 200-300 $\mu \mathrm{m}$ beyond the synaptic sites. In view of the fact that Xenopus myotomal muscle cells are innervated at their ends and are less than $200 \mu \mathrm{m}$ long (at the stages examined in this study), it is not surprising that in these cells the distribution of intracellular AChRs is essentially uniform.

At the onset of synaptogenesis in Xenopus myotomal muscle (Anderson and Cohen, 1977; Kuromi et al., 1985; Kidokoro et al., 1986), rat muscle (Ziskind-Conhaim et al., 1984), and chick muscle (Role et al., 1985), mobile AChRs in neighboring regions of the surface membrane enter and become immobilized in the newly forming postsynaptic membrane, thereby contributing to the build-up of the high AChR density. In rat and chick muscle (Kclly and Zacks, 1969; Nakai, 1968; sce also Englander and Rubin, 1987), but not in Xenopus myotomal muscle (Kullberg et al., 1977), this is followed by an accumulation of muscle nuclei immediately below the postsynaptic membrane. Subsynaptic nuclei provide a local source of mRNA for the synthesis of AChRs, which, in turn, must contribute to the maintenance of the high density of AChRs in the postsynaptic membrane (Merlie and Sanes, 1985; Fontaine et al., 1988). It is possible that the newly synthesized AChRs are inserted directly into the postsynaptic membrane or reach it by lateral movement after being inserted into perisynaptic surface membrane. The distributions of intracellular AChRs, and of perisynaptic surface AChRs, that have been observed so far are consistent with both possibilities not only in Xenopus myotomal muscle, which lacks subsynaptic nuclei, but also in rat and chick muscles, which contain subsynaptic nuclei. 


\section{References}

Anderson, M. J., and M. W. Cohen (1977) Nerve-induced and spontaneous redistribution of acetylcholine receptors on cultured muscle cells. J. Physiol. (Lond.) 268: 757-773.

Atsumi, S. (1981) Localization of surface and internal acetylcholine receptors in developing fast and slow muscles of the chick embryo. Dev. Biol. 86: 122-135.

Baldwin, T. J., C. M. Yoshihara, K. Blackmer, C. R. Kinter, and S. J. Burden (1988) Regulation of acetylcholine receptor transcript expression during development in Xenopus laevis. J. Cell Biol. 106: 469-478.

Betz, H., J.-P. Bourgeois, and J.-P. Changeux (1980) Evolution of cholinergic proteins in developing slow and fast skeletal muscle in chick embryo. J. Physiol. (Lond.) 302: 197-218.

Bevan, S., and J. H. Steinbach (1977) The distribution of $\alpha$-bungarotoxin binding sites on mammalian skeletal muscle developing in vivo. J. Physiol. (Lond.) 267: 195-213.

Blount, P., and J. P. Merlie (1988) Native folding of an acetylcholine receptor $\alpha$-subunit expressed in the absence of other receptor subunits. J. Biol. Chem. 263: 1072-1080.

Burden, S. (1977) Development of the neuromuscular junction in the chick embryo: The number, distribution and stability of acetylcholine receptors. Dev. Biol. 57: 317-329.

Carlin, B. E., J. C. Lawrence, Jr., J. M. Lindstrom, and J. P. Merlie (1986) An acetylcholine receptor precursor $\alpha$-subunit that binds $\alpha$-bungarotoxin but not $d$-tubocurare. Proc. Natl. Acad. Sci. USA 83 : 498-502.

Chow, I., and M. W. Cohen (1983) Developmental changes in the distribution of acetylcholine receptors in the myotomes of Xenopus laevis. J. Physiol. (Lond.) 339: 553-571.

Cohen, M. W., M. Greschner, and M. Tucci (1984) In vivo development of cholinesterase at a neuromuscular junction in the absence of motor activity in Xenopus laevis. J. Physiol. (Lond.) 348: 57-66.

Devreotes, P. N., and D. M. Fambrough (1976) Turnover of acetylcholine receptors in skeletal muscle. Cold Spring Harbor Symp. Quant. Biol. 40: 237-251.

Devreotes, P. N., J. M. Gardner, and D. M. Fambrough (1977) Kinetics of biosynthesis of the acetylcholine receptor and its subsequent incorporation into the plasma membrane of cultured chick skeletal muscle. Cell 10: 365-373.

Englander, L. L., and L. L. Rubin (1987) Acetylcholine receptor clustering and nuclear movement in muscle fibers in culture. J. Cell Biol. 104:87-95.

Fambrough, D. M., and P. N. Devreotes (1978) Newly synthesized acetylcholine receptors are located in the Golgi apparatus. J. Cell Biol. 76: 237-244.

Fambrough, D. M., P. N. Devreotes, and D. J. Card (1977) The synthesis and degradation of acetylcholine receptors. In Synapses, $\mathrm{G}$. A. Cottrell and P. N. R. Usherwood, eds., pp. 202-236, Blackie, Glascow.

Fontaine, B., D. Sassoon, M. Buckingham, and J.-P. Changeux (1988) Detection of the nicotinic acetylcholine receptor $\alpha$-subunit mRNA by in situ hybridization at neuromuscular junctions of 15-day-old chick striated muscle. EMBO J. 7: 603-609.

Gardner, J. M., and D. M. Fambrough (1979) Acetylcholine receptor degradation measured by density labeling: Effects of cholinergic ligands and evidence against recycling. Cell 16: 661-674.

Goldfarb, J., C. Cantin, and M. W. Cohen (1984) Developmental changes in the number of intracellular acetylcholine receptors in Xenopus myotomal muscle. Soc. Neurosci. Abstr. 10:581.

Harris, A. J. (1981) Embryonic growth and innervation of rat skeletal muscles. III. Neural regulation of junctional and extra-junctional acetylcholine receptor clusters. Phil. Trans. R. Soc. London [Biol.] 293: 287-314

Kelly, A. M., and S. I. Zacks (1969) The fine structure of motor endplate morphogenesis. J. Cell Biol. 42: 154-169.

Kidokoro, Y., B. Brass, and H. Kuromi (1986) Concanavalin A prevents acetylcholine receptor redistribution in Xenopus nerve-muscle cultures. J. Neurosci. 6: 1941-1951.

Kullberg, R., and H. Kasprzak (1985) Gating kinetics of nonjunctional acetylcholine receptor channels in developing Xenopus muscle. J. Neurosci. 5: 970-976.

Kullberg, R. W., T. L. Lentz, and M. W. Cohen (1977) Development of the myotomal neuromuscular junction in Xenopus laevis: An electrophysiological and fine-structural study. Dev. Biol. 60: 101-129.

Kullberg, R. W., F. S. Mikelberg, and M. W. Cohen (1980) Contribution of cholinesterase to developmental decreases in the time course of synaptic potentials at an amphibian neuromuscular junction. Dev. Biol. 75: 255-267.

Kullberg, R., J. L. Owens, and J. Vickers (1985) Development of synaptic currents in immobilized muscle of Xenopus laevis. J. Physiol. (I ond.) 364: 57-68

Kuromi, H., B. Brass, and Y. Kidokoro (1985) Formation of acetylcholine receptor clusters at neuromuscular junction in Xenopus cultures. Dev. Biol. 109: 165-176.

Lewis, P. R., and A. F. W. Hughes (1960) Patterns of myoneural junctions and cholinesterase activity in the muscles of tadpoles of Xenopus laevis. Q. J. Microsc. Sci. 101: 55-67.

Matthews-Bellinger, J., and M. M. Salpeter (1978) Distribution of acetylcholine receptors at frog neuromuscular junctions with a discussion of some physiological implications. J. Physiol. (Lond.) 279: 197-213.

McLean, I. W., and P. K. Nakane (1974) Periodate-lysine-paraformaldehyde fixative. A new fixative for immunoelectron microscopy. J. Histochem. Cytochem. 22: 1077-1083.

Merlie, J. P., and J. R. Sanes (1985) Concentration of acetylcholine receptor mRNA in synaptic regions of adult muscle fibers. Nature 317: 66-68.

Merlie, J. P., and M. M. Smith (1986) Synthesis and assembly of acetylcholine receptor, a multisubunit membrane glycoprotein. J. Membr. Biol. 91: 1-10.

Munt $<$, L. (1975) Myogenesis in the trunk and leg during development of the tadpole of Xenopus laevis (Daudin 1802). J. Embryol. Exp. Morphol. 33: 757-774.

Nakai, J. (1968) The development of neuromuscular junctions in cultures of chick embryo tissue. J. Exp. Zool. 170: 85-106.

Nieuwkoop, P. D., and J. Faber (1967) Normal Table of Xenopus laevis (Daudin), 2nd ed., North-Holland, Amsterdam.

Pestronk, A. (1985) Intracellular acetylcholine receptors in skeletal muscles of the adult rat. J. Neurosci. 5: 1111-1117.

Role, L. W., V. R. Matossian, R. J. O'Brien, and G. D. Fischbach (1985) On the mechanism of acetylcholine receptor accumulation at newly formed synapses on chick myotubes. J. Neurosci. 5: 2197-2204.

Steinbach, J. H. (1981) Developmental changes in acetylcholine receptor aggregates at rat skeletal neuromuscular junctions. Dev. Biol. 84: $267-276$.

Stollberg, J., and D. K. Berg (1987) Neuronal acetylcholine receptors: Fate of surface and internal pools in cell culture. J. Neurosci. 7: 18091815.

Ziskind-Conhaim, L., I. Geffen, and Z. W. Hall (1984) Redistribution of acetylcholine receptors on developing rat myotubes. J. Neurosci. 4: $2346-2349$. 